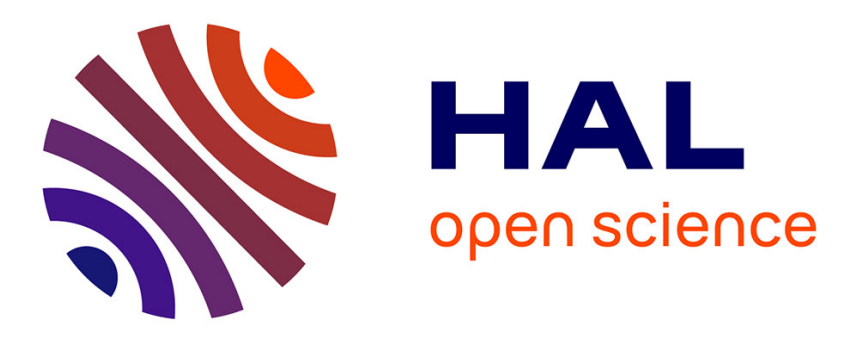

\title{
Numerical modeling of the effective ductile damage of macroporous alumina
}

Vincent Le Corre, Nadège Brusselle-Dupend, Maxime Moreaud

\section{To cite this version:}

Vincent Le Corre, Nadège Brusselle-Dupend, Maxime Moreaud. Numerical modeling of the effective ductile damage of macroporous alumina. Mechanics of Materials, 2017, 114, pp.161 - 171. 10.1016/j.mechmat.2017.08.002 . hal-01704402

\section{HAL Id: hal-01704402 https://hal-ifp.archives-ouvertes.fr/hal-01704402}

Submitted on 8 Feb 2018

HAL is a multi-disciplinary open access archive for the deposit and dissemination of scientific research documents, whether they are published or not. The documents may come from teaching and research institutions in France or abroad, or from public or private research centers.
L'archive ouverte pluridisciplinaire HAL, est destinée au dépôt et à la diffusion de documents scientifiques de niveau recherche, publiés ou non, émanant des établissements d'enseignement et de recherche français ou étrangers, des laboratoires publics ou privés. 


\title{
Numerical modeling of the effective ductile damage of macroporous alumina
}

\author{
Vincent LE CORRE $^{1 *}$, Nadège BRUSSELLE-DUPEND ${ }^{2}$, Maxime MOREAUD ${ }^{1,3}$
}

1 : IFP Energies nouvelles, Rond-point de l'échangeur de Solaize, BP 3, 69360 Solaize, France

2 : IFP Energies nouvelles, 1 et 4 avenue de Bois-Préau, 92852 Rueil-Malmaison, France

3: CMM - Centre de Morphologie Mathématique, Mines ParisTech, PSL Research University, Paris, France

* : corresponding author : vincent.le-corre@ifpen.fr

\begin{abstract}
:
Macroporous $\gamma$-alumina ceramics is a granular-like medium composed of agglomerated mesoporous grains allowing inter-granular macroporosity. Macropores, whose equivalent diameter is larger than $1 \mu \mathrm{m}$, represent a volume fraction of $14.5 \%$. The mechanical properties of the macroporous material are very low compared with the mesoporous matrix. In this work, the ductile damage mechanisms of the considered porous alumina are numerically investigated on a wider range of stress triaxiality ratio than experimentally thanks to a full-field homogenization approach based on a 2D Finite Element Method (FEM). The microstructure geometry is extracted from Scanning Electron Microscopy (SEM). The complexity of the macropores shape is closely transcribed from image treatment process to meshing. The matrix behavior is considered elastic-plastic and follows a Drucker-Prager yield surface. A reference plastic dissipation, adjusted on experimental uniaxial compression tests is used to approximate the plastic domain under negative mean stress. The calculated yield surface of the porous Drucker-Prager matrix presents a cap-form which is consistent with the shape identified experimentally. The overall yield surface is qualitatively the same cap-form as those obtained in literature for isotropic porous media containing spherical voids embedded in a Drucker-Prager matrix, which seems to show that the pore shape does not strongly affect this cap-form since the overall isotropy is respected.
\end{abstract}

Keywords : Alumina; porosity; non-convex pores; frictional cohesive material; ductile damage; Finite Element Method; digital image processing; full-field homogenization; Drucker-Prager yield surface; closed-form criterion

\section{Introduction, Porous $\gamma$-alumina solids}

Engineered porosity in ceramic materials extends their range of properties and offers promising performance for numerous applications. In the context of oil-refining, porous alumina ceramics are used as catalyst supports because of their large specific area - up to $250 \mathrm{~m}^{2} / \mathrm{g}$ - and of the possibility to control pore size and distribution. High mass transfer, low pressure drop and good heat management are essential features to obtain an efficient catalytic activity. The improvement of catalytic processes goes along with a general trend to increase the porous volume and the specific surface area of the supports, at the disadvantage of the mechanical stability. Mechanical strength is a key feature for catalysts supports. Catalytic activation, transport and in-service life generate various types of mechanical loading on catalyst grains, like multi-axial compression, traction induced by bending and shearing. Therefore, a fine understanding of the impact of the porosity on the fracture behavior of the supports under triaxial loads is needed to optimize the material design.

The present study focuses on hydro-treatment catalysts that are used in large fixed bed reactors of several meters high and wide. These solid catalysts are few millimeter long extrudates, composed of a polycrystalline $\gamma$-alumina phase forming a porous network on which a catalytic active phase is deposited. Depending on the process, two types of materials are used. Their textural properties, as obtained by mercury intrusion porosimetry, are presented in Table 1. Pore sizes are estimated thanks to the Young-Laplace equation relating the mercury pressure to a capillary radius (Lowell and Shields, 1991). The first material, noted mesoporous material, presents a monomodal and narrow pore size distribution centered under $10 \mathrm{~nm}$. The second material, noted macroporous material, presents a supplementary population of large pores which size can extent up to $10 \mu \mathrm{m}$. Figure 1 reveals the 
microstructure of both materials. Mesoporous material is composed of grains fully enclosed in a matrix, both made of mesoporous $\gamma$-alumina. Macroporous material is composed of mesoporous $\gamma$ alumina grains freely agglomerated, allowing intergranular macroporosity. As a consequence, macropores have complex shapes : cross-sections are frequently non-convex with multiple branches. Porosimetry analysis indicates that the macropores network is connected.

Table 1 : Textural properties of mesoporous and macroporous materials as obtained by mercury intrusion porosimetry

\begin{tabular}{cccc} 
& & \multicolumn{3}{c}{ Mesoporous material } & Macroporous material \\
\hline \multirow{2}{*}{$\begin{array}{c}\text { meso- } \\
\text { porosity }\end{array}$} & volume & $0.65 \mathrm{~mL} / \mathrm{g}$ & $0.65 \mathrm{~mL} / \mathrm{g}$ \\
\cline { 2 - 4 } & fraction & $66 \%$ & $55.5 \%$ \\
\cline { 2 - 4 } & pore size & $<10 \mathrm{~nm}$ & $<10 \mathrm{~nm}$ \\
\hline \multirow{2}{*}{$\begin{array}{c}\text { macro- } \\
\text { porosity }\end{array}$} & volume & - & $0.22 \mathrm{~mL} / \mathrm{g}$ \\
\cline { 2 - 4 } & fraction & - & $18 \%$ \\
\cline { 2 - 4 } & pore size & - & $0.4 \mu \mathrm{m}-10 \mu \mathrm{m}$ \\
\hline \hline
\end{tabular}

a)

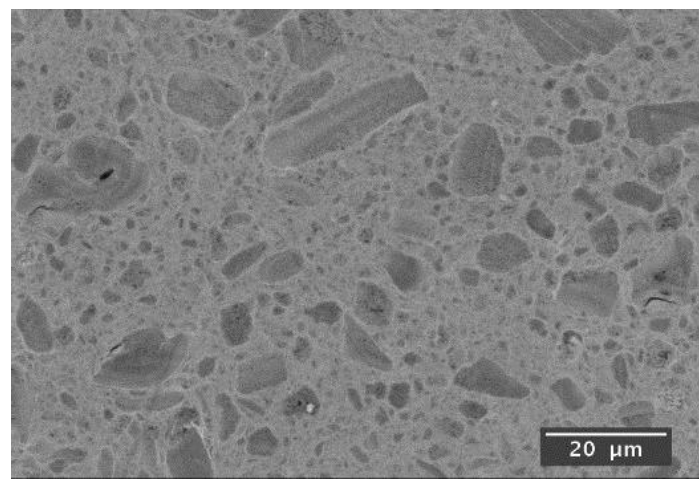

b)

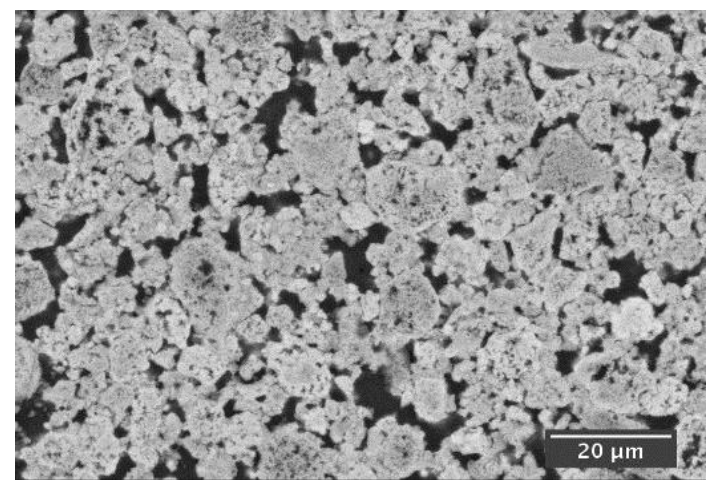

Figure 1 : Scanning electron microscopy of (a) mesoporous material and (b) macroporous material; backscattered electrons, polished surface after resin impregnation, porosity in black.

The mechanical behavior of $\gamma$-alumina catalyst supports has been characterized by (Staub et al., 2015; Staub et al., 2016). An elastic brittle behavior has been observed under tension. The macroporous $\gamma$ alumina exhibits a low mechanical strength compared with the mesoporous material (typically $8 \mathrm{MPa}$ against $17 \mathrm{MPa}$ in bending). A quasi-brittle behavior has been observed under compression and inelastic strains have been obtained under confined compression. Plasticity has been used to represent such a ductile damageable behavior under compression of both materials. Plastic yield surfaces have been identified and are detailed further. The macroporous material undergoes lower strength than the mesoporous material and residual macro-strains have been associated with a closure of the macroporosity. In order to improve mechanical strength of future materials, the role of the macroporosity has to be clarified.

The purpose of the study is to determine the impact of the complex macroporosity morphology on the damage mechanism and the global inelastic behavior, as there is no available criterion at the moment for this kind of porous material.

The major impact of porosity ratio on the mechanical strength of ceramics have been shown in pioneer work of (Duckworth, 1953). Since then numerous empirical relationships have then proposed to estimate the elastic stiffness and the axial strength of porous materials, see for instance (Wagh et al., 1993). Physical models have been developed and compared with a large experimental database, like in works of (Rice, 1993), based on the minimal surface area concept. Such concepts assume a periodic assembly of grains. These models mainly focus on elasticity and purely brittle behavior.

A first physical model of quasi-brittle behavior under uniaxial axisymmetric compression has been given by (Ashby and Sammis, 1990). Their model introduces stability and damage notions based on fracture mechanics concepts and does not explicitly involve porosity. More recently, the pore-pore 
interactions and their impact on fracture have been investigated from several approaches. An increase of damage dissipation with porosity has been observed in lightweight concrete by (Miled et al., 2004) and an analytical model was developed to predict its quasi-brittle behavior. The dominating fracture mechanism was determined by comparison of the local fracture process zone with a characteristic length of the microstructure. A transition from brittle to quasi-brittle behavior has also been observed by (Meille et al., 2012) under uniaxial compression as porosity increases from $30 \%$ to $75 \%$. The porosity at which the transition occurs, around $60 \%$, is estimated by a simple model taking into account the competition between the crack length initiating from spherical pores and the mean distance between pores. The model thus explains why pore size distribution and spatial homogeneity acts upon fracture mechanisms by modifying the wall size between pores.

In addition to the description of the fracture under uniaxial tension and compression, a lot of studies in literature deal with the determination of multiaxial constitutive laws for these granular-like porous materials. Micromechanical homogenization models based on mean fields approach have brought a new and promising frame (Bornert et al., 2001). Porosity morphology can be taken into account through the aspect ratios of ellipsoidal pores or ellipsoidal grains. Pore size distribution does not have an influence as soon as the scale separation assumption is respected. Macroscopic criteria have been obtained in case of spherical pores in a matrix for numerous types of plastic behaviors (Barthélémy and Dormieux, 2003). Granular cohesive behavior of polycrystal materials have also been estimated by homogenization of a medium composed of ellipsoidal elastic inclusions joined by brittle or ductile interfaces (Maalej et al., 2009; Sanahuja et al., 2010). Numerous types of pressure sensitive behavior of the matrix can be taken into account, e.g. (Shen et al., 2015). Moreover some studies combine classical fracture mechanisms approach or limit analysis of hollow sphere obeying a plastic criterion with FE simulation to search for macroscopic criterion, e.g. (Thoré et al., 2009). These methods lead to multiaxial behavior laws. The impact of porosity ratios is successfully reproduced when pores can be considered as ellipsoids. However, no equivalence exists to translate a complex porosity shape into ellipsoidal shape ratio. This gap is particularly detrimental to determine macroscopic properties if the solid phase is close to the loss of percolation and the mechanical strength strongly decreases .

As an alternative to micromechanical models, direct numerical simulation aims at deriving complete constitutive laws with minimum assumptions about the morphology of microstructures. Such techniques have extensively been developed in recent years thanks to the improvement of observation techniques and the growth of computing capacity. The geometry of the microstructure, determined by digitization of observations, can be used directly in simulations. Or, morphological models can be adjusted on observations in order to simplify numerical models construction (Jeulin, 2001). Numerical homogenization of porous material behavior can be carried out by several techniques. Discrete Element Methods (DEM) seem naturally adapted for cohesive granular materials (Jauffrès et al., 2012). However, the representation of the porosity morphology and of the grains shape is limited. The homogenization problem has also been addressed using periodic full fields approaches solved thanks to a Fast Fourier Transformation (FFT) method developed by (Moulinec and Suquet, 1998), later modified for porous materials by (Michel et al., 2001). FFT methods advantageously use 2D or 3D images of the microstructure to describe the geometry of phases. Pressure-sensitive plasticity models needs to be further implemented to be applied to granular materials. Eventually, the Finite Element Method (FEM) remains broadly used for direct numerical homogenization. This method becomes computationally affordable when combined with morphological models that helps meshing operation thanks to analytic descriptions of geometries (Ayyar and Chawla, 2006; Dirrenberger et al., 2014; Fritzen et al., 2012; Keleş et al., 2013; Khdir et al., 2014; Shen and Brinson, 2007). By combining 2D FE simulations and classical fracture mechanics, (Keles et al., 2013) have evidenced the influence of stress triaxiality on the fracture strength of brittle porous materials. More recently, image processing techniques have been combined with meshing tools to allow Digital Image Based Finite Element Analysis (DIB-FEA). This method has been proved reliable to estimate the elastic and plastic properties of materials, for instance ceramics obtained by gel casting of yttria partially stabilized zirconia powders (Bartuli et al., 2009), cellular ceramics (Petit et al., 2013), stainless steels (Dancette et al., 2016) or sintered steels (Chawla and Deng, 2005). In this last example, simulation demonstrates how irregular and highly clustered pores contribute to significant strain localization and early failure. 
The choice of FEM is relevant when driven by the will to address highly non-linear problems with a fine description of the morphology.

In the next section, the macroscopic mechanical properties of porous $\gamma$-alumina materials used as catalyst supports are shown. A numerical finite element analysis (FEA) based on a real microstructure acquired from scanning electron microscopy (SEM) is then presented in order to assess the impact of macro-porosity on the global behavior. The macroporous material is modeled as a mesoporous matrix with realistic non-convex macropores. Homogenized macroporous behavior is then compared with experimental data and the results are finally discussed.

\section{Macroscopic behavior of meso and macroporous $\gamma$-alumina}

The macroscopic behavior of $\gamma$-alumina catalyst supports has been characterized by Staub et al. (Staub et al., 2015; Staub et al., 2016), thanks to series of laboratory tests. Tensile strengths $\sigma_{t}$ have been determined from bending tests. Dispersion of brittle fracture strength under tension can be expressed by a Weibull distribution of the fracture probability $\mathrm{P}_{\mathrm{F}}$ :

$$
\mathrm{P}_{\mathrm{F}}=1-\exp \left[-\left(\frac{\sigma_{\mathrm{t}}}{\mathrm{A}}\right)^{\mathrm{m}}\right]
$$

Eq. 1

where $\mathrm{m}$ is the Weibull modulus and $\mathrm{A}$ is reference stress, given in Table 2 together with the mean tensile strength $\overline{\sigma_{t}}$.

A ductile damageable behavior under compression has been characterized by instrumented spherical indentation tests and plastic yield surfaces have been estimated. Both materials exhibit a sensibility of the strength to the mean stress. Such a frictional cohesive behavior is coherent with the granular microstructure of these materials, like rocks or concrete. As promoted by works of (Rudnicki and Rice, 1975) for brittle rock, a plasticity model has been used by (Staub et al., 2016) to describe the inelastic behavior likely produced by nucleation and growth of micro-cracks and by frictional sliding of the micro-crack surfaces. The monomodal mesoporous alumina ductile damage criterion was shown to be represented by a Drucker-Prager yield surface (Drucker, 1966) :

$$
\sigma_{\text {eq }}+\tan \left(\beta_{\text {meso }}\right) \cdot \sigma_{\mathrm{m}}-\mathrm{d}_{\text {meso }}=0
$$

where $\sigma_{\mathrm{m}}$ is the mean stress of the Cauchy stress tensor $\sigma: \sigma_{\mathrm{m}}=\frac{1}{3} \operatorname{tr}(\sigma), \sigma_{\mathrm{eq}}$ is the equivalent stress of von Mises : $\sigma_{\mathrm{eq}}=\sqrt{\frac{3}{2} \cdot \mathrm{s} \text { : } \mathrm{s}}$ with s the deviatoric stress tensor $\mathrm{s}=\sigma-\sigma_{\mathrm{m}}$. I and I the $2^{\text {nd }}$ order identity tensor, $\beta_{\text {meso }}$ is the internal friction angle and $\mathrm{d}_{\text {meso }}$ is the cohesion of the material.

As concerns macroporous alumina, the fracture criterion was shown to be represented by a DruckerPrager yield surface closed by a cap surface that expresses the ability of the material to admit plastic compaction for high pressure loads, as observed experimentally by the closure of macropores:

$$
\sqrt{\left[\sigma_{\mathrm{m}}-\sigma_{\mathrm{m} 1}\right]^{2}+\left[\mathrm{R} \cdot \sigma_{\mathrm{eq}}\right]^{2}}-\text { R. }\left[\mathrm{d}_{\mathrm{macro}}-\sigma_{\mathrm{m} 1} \cdot \tan \left(\beta_{\mathrm{macro}}\right)\right]=0
$$

where $\sigma_{\mathrm{m} 1}$ is the mean stress above which plastic compaction can occur $\left(\sigma_{\mathrm{m} 1}<0\right), \mathrm{R}$ is the shape factor of the cap, a quarter of ellipse in the meridional plane $\left(\sigma_{\mathrm{m}}, \sigma_{\mathrm{eq}}\right)$. R can be related to the yield pressure in pure hydrostatic compression $\sigma_{\mathrm{m} 2}$ :

$$
\mathrm{R}=\frac{\sigma_{\mathrm{m} 1}-\sigma_{\mathrm{m} 2}}{\mathrm{~d}_{\text {macro }}-\sigma_{\mathrm{m} 1} \cdot \tan \left(\beta_{\text {macro }}\right)}
$$

All material behavior parameters are reported in Table 2. Under uniaxial tension, macroporous $\gamma$ alumina exhibits low mechanical strength compared with the mesoporous material (typically $8 \mathrm{MPa}$ against $17 \mathrm{MPa}$ in bending). Under compression, the experimental results reveal that the cohesion of the macroporous alumina is twice lower than the cohesion of the mesoporous alumina (17 MPa against $30 \mathrm{MPa}$ ). Otherwise, a plastic compaction has been estimated on macroporous material for pressures higher than $21.5 \mathrm{MPa}$, whereas compaction has not been detected on mesoporous material under pressures up to $28 \mathrm{MPa}$. By comparison with the mesoporous material, the low mechanical strength of 
the macroporous material might be caused by macroporosity. The size and the morphology of the macropores is suspected to play an essential role. In the next section, a full-field homogenization by FEM based on SEM images of the porous microstructure of macroporous alumina is set up in order to better understand the impact of the macroporosity on the macroscopic inelastic behavior and on the local damage mechanisms.

Table 2 : Material behavior parameters as identified in (Staub, 2014), (1) from compressive tests on cylinders, (2) from 3-points-bending tests, (3) from spherical indentation tests

\begin{tabular}{cccccccccc} 
& \multicolumn{3}{c}{ Elasticity (1) } & \multicolumn{3}{c}{ Tensile strength (2) } & \multicolumn{3}{c}{ Compressive strength (3) } \\
\cline { 2 - 9 } & $\begin{array}{c}\mathrm{E} \\
{[\mathrm{GPa}]}\end{array}$ & $v$ & $\begin{array}{c}\overline{\sigma_{\mathrm{t}}} \\
{[\mathrm{MPa}]}\end{array}$ & $\begin{array}{c}\mathrm{A} \\
{[\mathrm{MPa}]}\end{array}$ & $\mathrm{m}$ & $\begin{array}{c}\mathrm{d} \\
{[\mathrm{MPa}]}\end{array}$ & $\beta[\mathrm{deg}]$ & $\begin{array}{c}\sigma_{\mathrm{m} 1} \\
{[\mathrm{MPa}]}\end{array}$ & $\begin{array}{c}\sigma_{\mathrm{m} 2} \\
{[\mathrm{MPa}]}\end{array}$ \\
\hline $\begin{array}{c}\text { Mesoporous } \\
\text { Alumina }\end{array}$ & 5.7 & 0.22 & 17.1 & 18 & 7.5 & 30 & 43 & $/$ & $/$ \\
\hline $\begin{array}{c}\text { Macroporous } \\
\text { Alumina }\end{array}$ & 2.5 & 0.22 & 8.1 & 9 & 8.9 & 17 & 13 & 21.5 & 26 \\
\hline \hline
\end{tabular}

\section{Full-field homogenization based on FEM and SEM digital images}

\subsection{Digital images processing}

The macroscopic properties obtained in the homogenization approach are valid if the sample is a Representative Volume Element (RVE) of the material for the property of interest. Alumina macropores range from $0.05 \mu \mathrm{m}$ to $10 \mu \mathrm{m}$. Representative samples of the microstructure are larger than $100 \mu \mathrm{m}^{3}$. Laboratory $\mathrm{x}$-ray computed tomography can generate 3D images of such volume with a resolution around $1 \mu \mathrm{m}$. However, noise is too high at this resolution to catch fine details of the pores geometry. High resolution tomography, as with synchrotron $\mathrm{x}$-ray sources, would be appropriate; however such data is not available so far on the material of the study. Focused Ion Beam (FIB) cutting coupled to SEM also provides 3D images with excellent resolution, around $10 \mathrm{~nm}$. The resolution obtained with this technique is gradually worsened by curtaining effect due to porosity and final sampled volumes are too small to be representative of the microstructure. Finally, 2D SEM images of microstructure cross-section offer a good compromise. The porosity surface fraction can be considered equal to the pore volume fraction under stationary and ergodicity assumptions. Only the apparent pore size is altered by the 2D sampling. The resolution is well adapted to reveal the complexity of the porosity morphology and to sample a RVE. This technique was thus selected for the present study.

The resolution of SEM images used for the analysis was $450 \mathrm{~nm} /$ pixel. Square images of $100 \mu \mathrm{m}$, $200 \mu \mathrm{m}$ and $300 \mu \mathrm{m}$ were sampled. Samples were processed with a Flowing Bilateral Filter (Moreaud and Cokelaer, 2015) to reduce noise, then segmented in two phases thanks to a threshold gray level. The threshold was obtained by maximization of interphase variance (Otsu, 1979). Pores lower than 4 pixels i.e., pores whose geometry was poorly described, were removed for simplification. The pore surface fraction obtained by this segmentation was around $14.5 \%$. As a consequence, a pore volume fraction estimated around 3.5\% was not taken into account in the analysis. It corresponds to pores smaller than $0.9 \mu \mathrm{m}$. Figure 2 shows the SEM image sample and the segmented image resulting from the image processing.

The covariance $C(X, h)$ gives the probability of 2 points in an image at a distance $h$ to be in the same phase $X$, (Matheron, 1972). The distance $h_{c}$ for which $C\left(X, h_{c}\right)=C(X, 0)^{2}$ is a characteristic size for the phase $\mathrm{X}$. Figure 3 shows the covariance of the porosity phase in the segmented image. As the image orientation had been chosen randomly and horizontal and vertical covariances are identical, the microstructure can be considered isotropic. The characteristic pore size obtained on this image of size of $200 \times 200 \mu \mathrm{m}^{2}$ was close to $5 \mu \mathrm{m}$. For a Boolean scheme of spheres, RVE for calculation of elastic properties is reached for a volume greater than 8 times the average size of a sphere (El Moumen et al., 2013). Here the volume is 40 times greater than the average inclusion size, so we can consider almost 
surely that the images are representative of the morphology of the porosity for the calculation of elastic mechanical properties.

The contours of pores were extracted from the segmented image as a list of coordinates of pixels at boundaries. At this step, pores are polygones that can have sharp angles because of limited resolution. Higher resolution images have proven that these sharp angles are not realistic. So, the pore contours were smoothed to improve the relevance of the geometry transcription. Each point coordinate $x_{i}$ was weighted by its neighbors to obtain smoothed coordinate $x_{i}{ }_{i}: x_{i}^{\prime}=\frac{x_{i-1}+2 x_{i}+x_{i+1}}{4}$. The porosity fraction could be slightly affected by this operation. In this case, a homothetic growth around the pore barycenter restored the initial porosity. The smoothed contours points were used to re-build the geometry of the porous matrix in pre-processing software Abaqus/CAE®.

a)

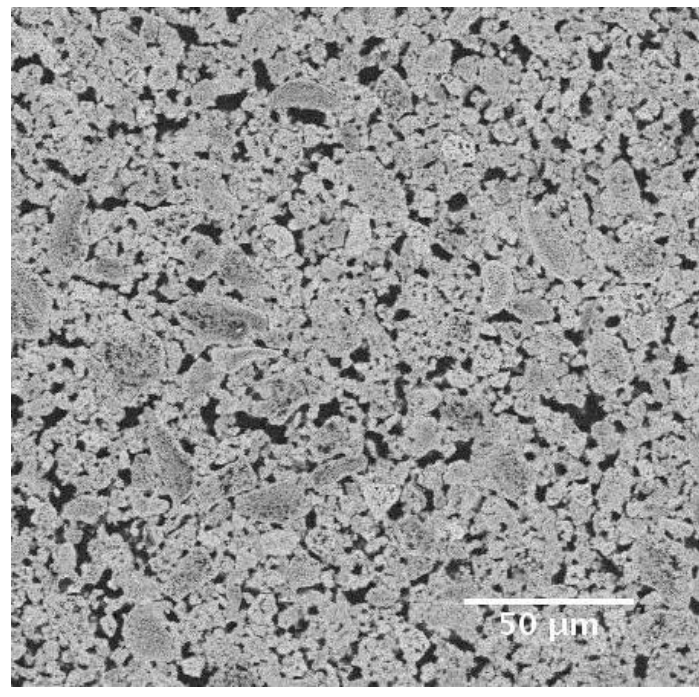

b)

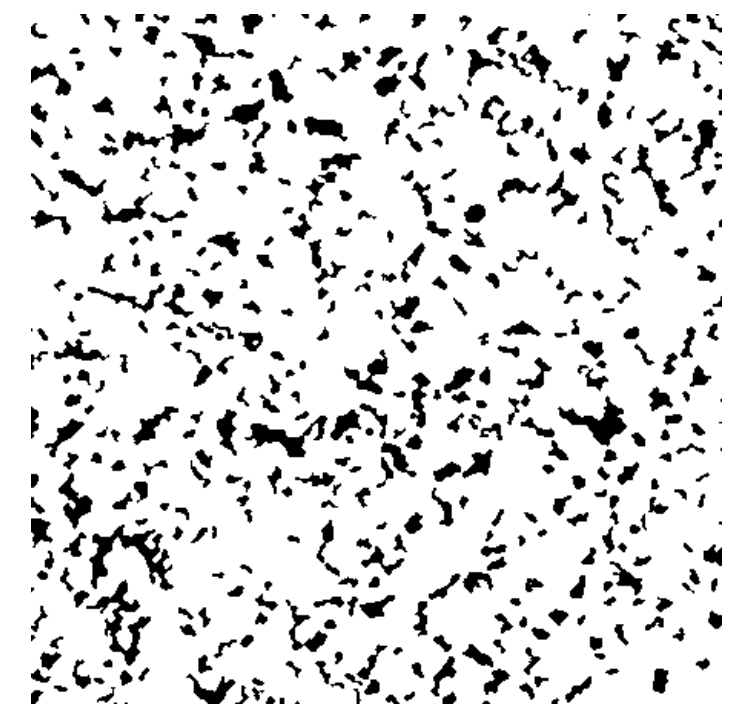

Figure 2 : a) Sample of SEM observations of a macroporous $\gamma$-alumina, 200x200 $\mu \mathrm{m}$ b) Segmented image (porosity in black)

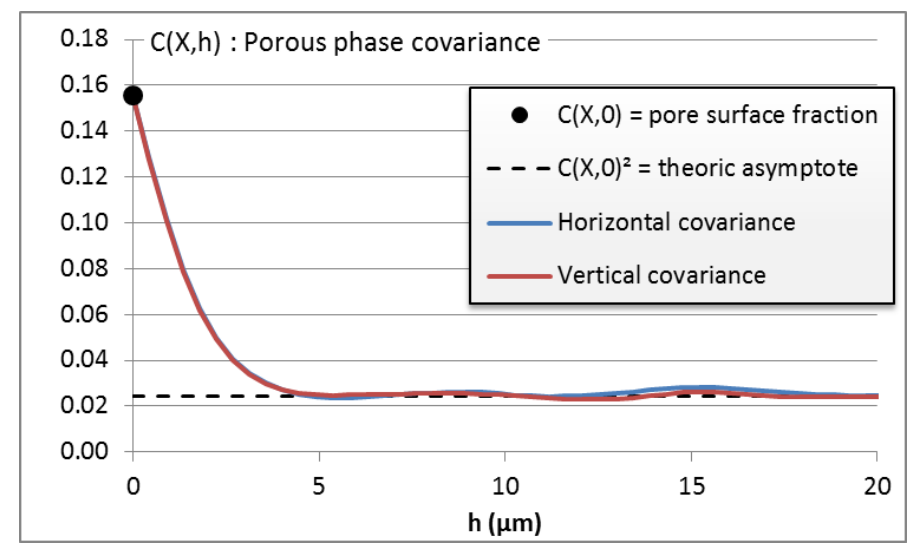

Figure 3 : Covariance of the porous phase in segmented SEM image

\subsection{Mesh generation}

Abaqus ${ }^{\circledR}$ Finite Element package was used to generate meshing and to achieve simulations. Second order triangular elements were used with a generalized plane strain formulation. A basic analysis has shown that the macroscopic behavior did not significantly depend on the characteristic mesh size. However, very fine meshes produced early divergence of simulations because of excessive local strains in small elements. The chosen characteristic element size was finally close to the pixel size of the SEM image used to build the geometry. Figure 4 illustrates a detail of the mesh. For example, 230000 elements were typically generated for a $200 \times 200 \mu \mathrm{m}^{2}$ image. 


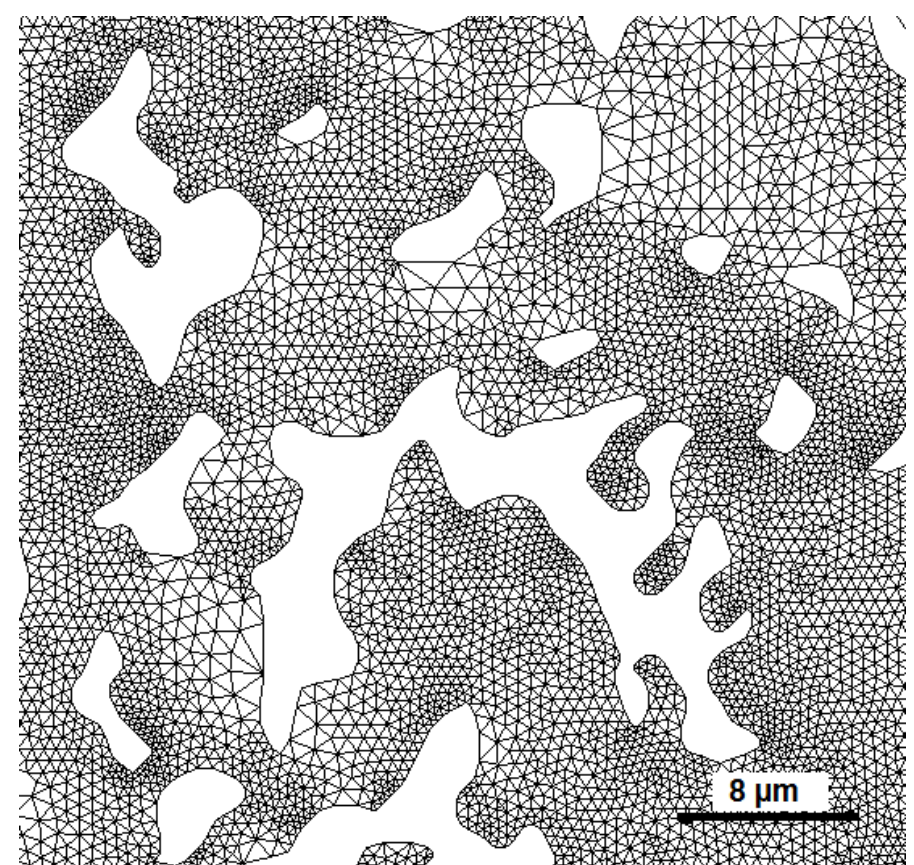

\section{Figure 4 : Detail of solid matrix meshing around a large pore; geometry based on pores contours extracted from segmented SEM image}

\subsection{Numerical Model}

\subsubsection{D formulation}

As explained in introduction of the paper, direct 3D simulations were not possible since the porosity geometry was not available in 3D. By default, 2D simulations poorly estimate the out-of-plane behavior. In basic plane stress and plane strain formulations, only a narrow range of triaxiality values are accessible since macro stress $\Sigma_{33}$ is not controlled. The generalized plane strain formulation was chosen in this study since it allowed to investigate all stress triaxiality ratios and thus to estimate the ductile damage onset in a $\left(\Sigma_{\text {eq }} ; \Sigma_{\mathrm{m}}\right)$ plane.

\subsubsection{Boundary conditions}

Solving the homogenization problem consists in obtaining the relation between the macroscopic strain and stress tensors, by imposing one and calculating the other. By definition, these tensors are equal to the average of the microscopic tensors. The average values can be controlled by imposing values on the contour of the domain only, i.e., by imposing boundary conditions. Numerous types of boundary conditions sets have been evaluated for homogenization issues. It has been shown that they have an impact on the homogenized macroscopic behavior if the sample volume is smaller than the RVE (Hazanov and Huet, 1994). Kinematic uniform boundary conditions (KUBC), obtained by artificially constraining nodes displacements, naturally tend to over-estimate the macroscopic stiffness. Static uniform boundary conditions tend to under-estimate stiffness by assuming absolutely no kinematic influence of the exterior media. Boundary conditions that mix static and kinematic constraints offer good compromise between these two limits. Periodic boundary conditions also provide interesting results since the shape of the period is free, so the kinematic constraint is lower than in pure KUBC. However, this method requires a periodic domain. In the present study, mix boundary conditions were used : on each face, the normal displacement was uniform and the tangential displacement was free. As a consequence, the macroscopic tangential component of the stress vector was zero on each face. Uniform normal displacement was obtained by imposing a kinematic coupling on each face between an auxiliary node and all the nodes of the face. Similarly, by definition of the generalized plane strain conditions, an auxiliary point was used to control the out-of-plane strain of the entire domain. Depending on the loading case, the auxiliary points were displacement-controlled or load-controlled. The macroscopic stress components were calculated by dividing the forces at auxiliary nodes by the face area. 


\subsubsection{Matrix behavior}

The matrix behavior was supposed to match the mesoporous material behavior. According to experimental characterization, the mesoporous material obeys a ductile damageable behavior under compression, likely produced by nucleation and growth of micro-cracks and by frictional sliding of the micro-crack surfaces. Plasticity was favored to represent ductile damage in the simulation whatever the load case, even if the failure mechanisms are brittle-like under tension. So, only non-linear effects due to plasticity were simulated.

The plastic behavior of the matrix was thus characterized by a Drucker-Prager yield surface whose parameters are given in Table 2, with associated plastic flow and no hardening. Self-contact was taken into account in case of pore closure. Post-processing showed that this situation is very rarely encountered.

\subsubsection{Applied loads}

Two sets of simulations were performed to estimate the effective elastic and inelastic properties of the macroporous material.

The first set of simulations aimed at assessing the macroscopic elastic tensor $\mathbb{C}^{\text {hom }}$ of the macroporous material, such that $\Sigma=\mathbb{C}^{\text {hom }}: E$, with $\Sigma$ and $E$ respectively the macroscopic stress and strain tensors, equal to the average of the local stress and strain tensors in the RVE. We only focused on the in-plane components of the tensor. Three elementary macroscopic strains $\mathrm{E}$ were applied to the domain by imposing uniform displacements of faces. In Voigt notation :

- $\quad$ an axial strain of $1 \%$ in direction 1, i.e. $\mathrm{E}=0.01 \cdot(1,0,0,0,0,0)$

- $\quad$ an axial strain of $1 \%$ in direction 2, i.e. $\mathrm{E}=0.01 \cdot(0,1,0,0,0,0)$

- $\quad$ a shear strain of $1 \%$ in the $(1 ; 2)$ plane, i.e. $E=0.01 \cdot(0,0,0,0,0,1)$

Simulations were achieved under linear static analysis, assuming small displacements and strains. For these load cases, the matrix was assigned a purely elastic behavior, with elastic properties of the mesoporous material, Table 2.

The second set of simulations aimed to assess the macroscopic ductile behavior of the macroporous material. Series of loads were applied in order to explore the RVE behavior in all directions of the $\left(\Sigma_{\text {eq }} ; \Sigma_{\mathrm{m}}\right)$ plane, i.e. with different values of stress triaxiality ratio. Normal forces were applied to domain faces through the auxiliary points. The macroscopic stress tensor $\Sigma$ was imposed with following features :

$$
\Sigma=\left(\begin{array}{lll}
1 & 0 & 0 \\
0 & \alpha & 0 \\
0 & 0 & \alpha
\end{array}\right) \cdot \dot{\Sigma} \cdot \mathrm{t}
$$

Eq. 5

with t the virtual time, time increment being automatically adapted as a function of convergence quality, $\dot{\Sigma}$ the stress rate, equal to $-40 \mathrm{MPa} . \mathrm{s}^{-1}$ for compressive loads and $40 \mathrm{MPa} . \mathrm{s}^{-1}$ for tensile loads and $\alpha$ a scalar parameter driving the triaxiality ratio, adopting values in range $[-0.5,1]$. The out-ofplane macro stress $\Sigma_{33}$ was deliberately chosen equal to $\Sigma_{22}$ in order to simplify the parameterization.

Such a parameterization led to following expressions of mean stress $\Sigma_{\mathrm{m}}$ and equivalent von Mises stress $\Sigma_{\text {eq }}$ :

$$
\begin{aligned}
& \Sigma_{\mathrm{m}}=\frac{1}{3}(1+2 \alpha) \cdot \dot{\Sigma} \cdot \mathrm{t} \\
& \Sigma_{\text {eq }}=|(1-\alpha) \cdot \dot{\Sigma}| \cdot \mathrm{t}
\end{aligned}
$$

and the macroscopic stress triaxiality ratio $\tau$ could be written as follows :

$$
\tau=\Sigma_{\mathrm{m}} / \Sigma_{\text {eq }}=\frac{1+2 \alpha}{3 \cdot|1-\alpha|} \cdot \operatorname{sign}(\dot{\Sigma})
$$

Thus, for $\alpha=0$, the applied load corresponded to a pure traction or compression. For $\alpha=1$, the applied load corresponded to a hydrostatic traction or compression. For $\alpha=-0.5$, the applied load corresponded to a pure deviatoric macro-stress.

Moreover, the macroscopic volumic strain $\mathrm{E}_{\mathrm{vol}}$ and equivalent strain $\mathrm{E}_{\mathrm{eq}}$ were computed as follows : 


$$
\begin{aligned}
& E_{v o l}=E_{11}+E_{22}+E_{33} \\
& E_{e q}=\sqrt{2} \cdot \sqrt{\left(E_{11}-E_{22}\right)^{2}+\left(E_{22}-E_{33}\right)^{2}+\left(E_{33}-E_{11}\right)^{2}}
\end{aligned}
$$

and the plastic components were deduced by subtracting the elastic parts :

$$
\begin{aligned}
& \mathrm{E}_{\mathrm{vol}}^{\mathrm{pl}}=\mathrm{E}_{\mathrm{vol}}-\frac{\Sigma_{\mathrm{m}}}{\mathrm{K}} \\
& \mathrm{E}_{\mathrm{eq}}^{\mathrm{pl}}=\mathrm{E}_{\mathrm{eq}}-\frac{\Sigma_{\mathrm{eq}}}{\mathrm{G}}
\end{aligned}
$$

where $\mathrm{K}$ and $\mathrm{G}$ are respectively the effective elastic bulk and shear modulus measured at the first increment of each simulation, as the plastic strain is zero.

Simulations were carried out with large displacement and large strain assumptions. An implicit integration scheme was used to solve iteratively the mechanical equilibrium at each time increment. The simulation ended if convergence was not achieved within a time increment lower than $10^{-5} \mathrm{~s}$ corresponding to a stress increment lower than $0.4 \mathrm{kPa}$.

\section{$4 \quad$ Numerical results}

\subsection{Elasticity}

Considering elementary macro strain $\mathrm{E}$ imposed in each elastic simulation, the components of the macroscopic elastic tensor $\mathbb{C}^{\text {hom }}$ were easily deduced from the stress tensors $\Sigma$ obtained numerically. The in-plane components of the tensor in Voigt notation are given below and compared with the experimental values computed with elastic constants given by (Staub et al., 2016) :

$$
\mathbb{C}_{2 \mathrm{D}}^{\text {hom }}=\left|\begin{array}{ccc}
2.889 & 0.696 & 0 \\
0.696 & 2.819 & 0 \\
0 & 0 & 0.677
\end{array}\right| \mathrm{GPa} \quad \mathbb{C}_{2 \mathrm{D}}^{\exp }=\left|\begin{array}{ccc}
2.837 & 0.624 & 0 \\
0.624 & 2.837 & 0 \\
0 & 0 & 0.553
\end{array}\right| \mathrm{GPa}
$$

The tensor is close to isotropy since the difference between components $\mathrm{C}_{11}$ hom and $\mathrm{C}_{22}{ }^{\text {hom }}$ is less than $2.5 \%$ and the microstructure is isotropic. The homogenized values are close to the experimental elastic tensor of the macroporous material : differences between homogenized and experimental values of $\mathrm{C}_{11}$ and $\mathrm{C}_{22}$ is less than $2 \% . \mathrm{C}_{12}{ }^{\text {hom }}$ and $\mathrm{C}_{66}{ }^{\text {hom }}$ are respectively overestimated by $11 \%$ and $22 \%$. For a Poisson's ratio $v^{\text {hom }}=0.22$, a typical value for highly porous materials (Sanahuja et al., 2010), the Young's modulus minimizing the difference - at least square sense - between the homogenized tensor and an isotropic tensor is $\mathrm{E}^{\mathrm{hom}}=2.74 \mathrm{GPa}$, in good agreement with the experimental value $\mathrm{E}^{\text {exp }}=2.5$ $\mathrm{GPa}$. The difference might be attributed to the omission of pores smaller than $0.9 \mu \mathrm{m}$ in the image processing.

\subsection{Ductile behavior under compressive loads $(\dot{\Sigma}<0)$}

\subsubsection{Focus on the ductile behavior under uniaxial compression}

Figure 5 presents the macroscopic behavior of the macroporous material under uniaxial compression. Simulation results are compared with experimental quasi-brittle behavior observed in (Staub et al., 2016). The simulation slightly over-estimates the elastic stiffness. The second graphic of Figure 5 focuses on the plastic behavior, the elastic part of strain is removed on each curve. The progressive loss of tangent stiffness due to ductile damage is satisfactorily reproduced by the plastic model. The brittle instability, corresponding to a macro crack propagation in the sample, is obviously not obtained by the simulation since no brittle damage model is implemented in the matrix behavior. The simulation only reproduces a ductile damage mechanism that produces hardening before global fracture. The plastic dissipation starts for an axial compressive stress of $10 \mathrm{MPa}$.

As illustrated in Figure 6, plasticity activates in stress concentration zones at tips of pores branches, then extends along bands linking neighboring pores. In Figure 7, the equivalent plastic strain field is superimposed on the microstructure picture used to obtain the RVE mesh. The plasticity is localized in areas that can be compared to grains interfaces, which is consistent with the inter-granular fracture revealed by SEM fractography in Figure 8. 

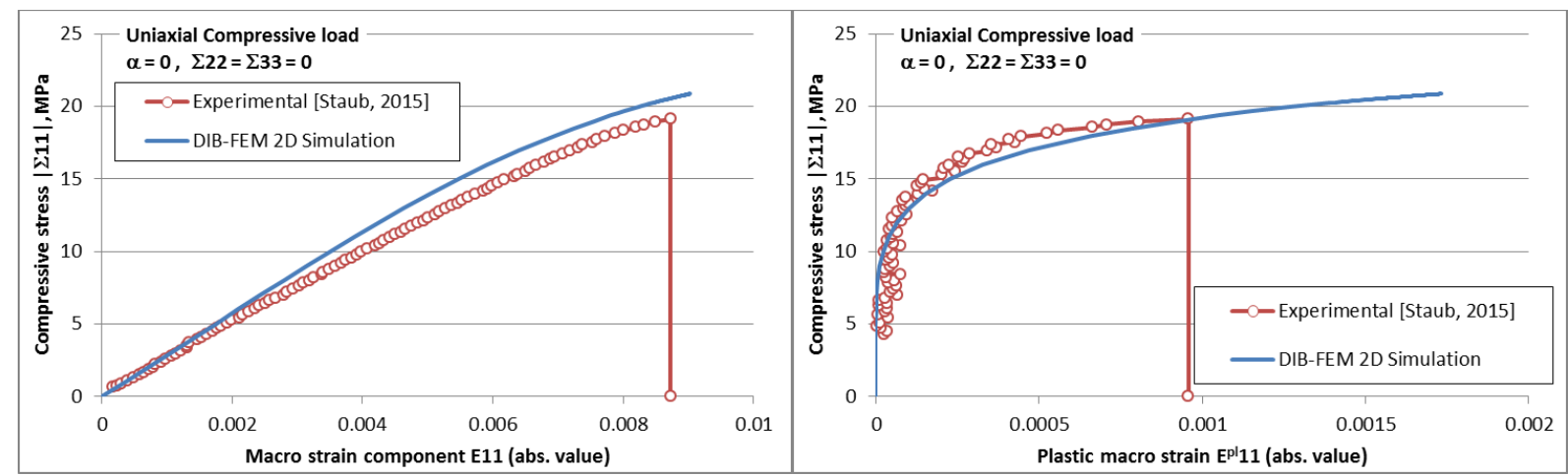

Figure 5 : Comparison of experimental and numerical macroporous material behavior under uniaxial compression, elastic-plastic strain (left) and plastic strain (right).
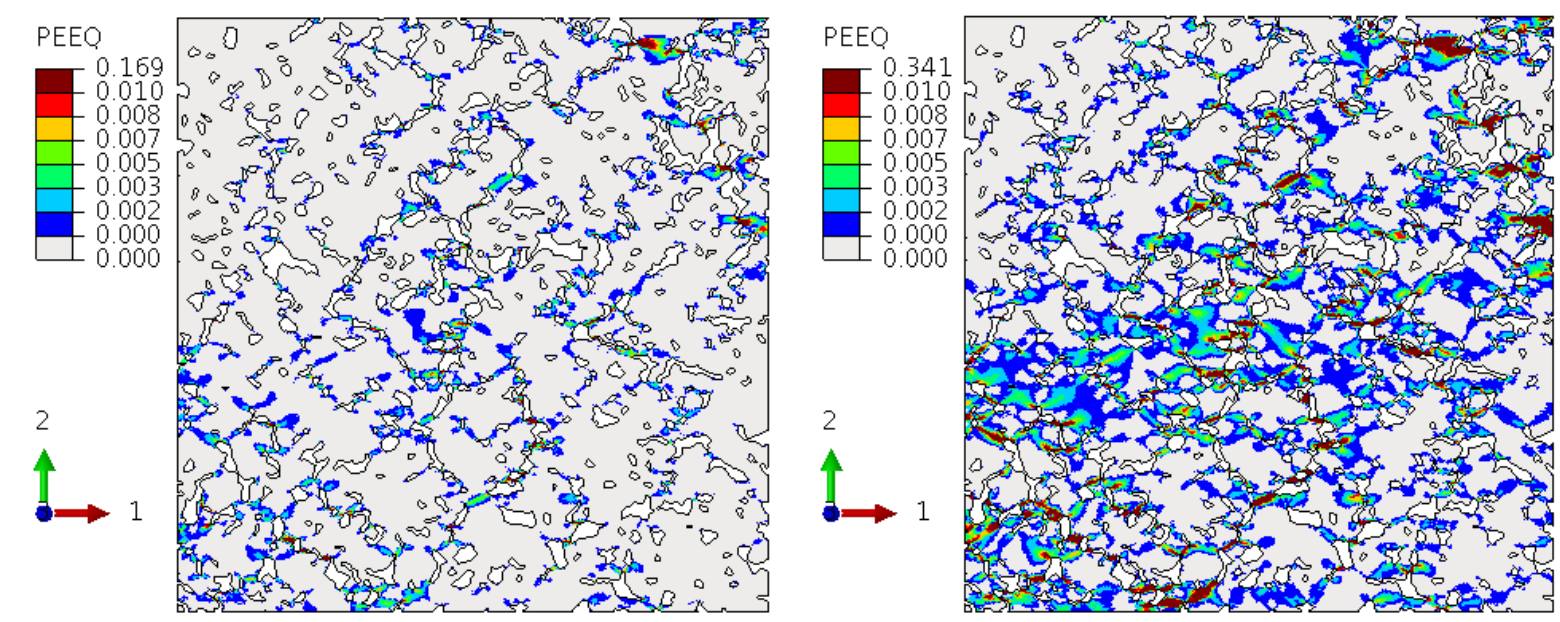

Figure 6 : Equivalent plastic strain field under uniaxial compression for $\Sigma_{11}=-20 \mathrm{MPa}$ (left) and at end of simulation for $\Sigma_{11}=-24 \mathrm{MPa}$ (right)

a)

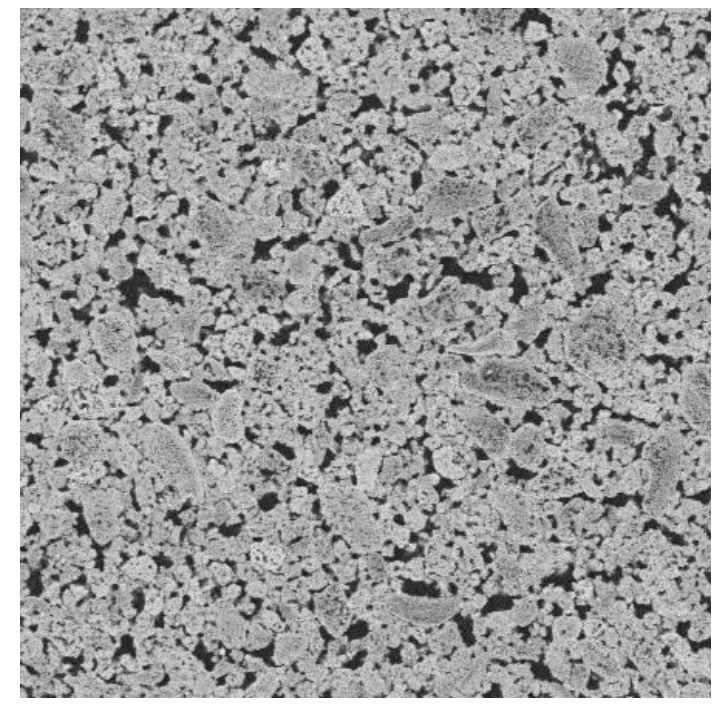

b)

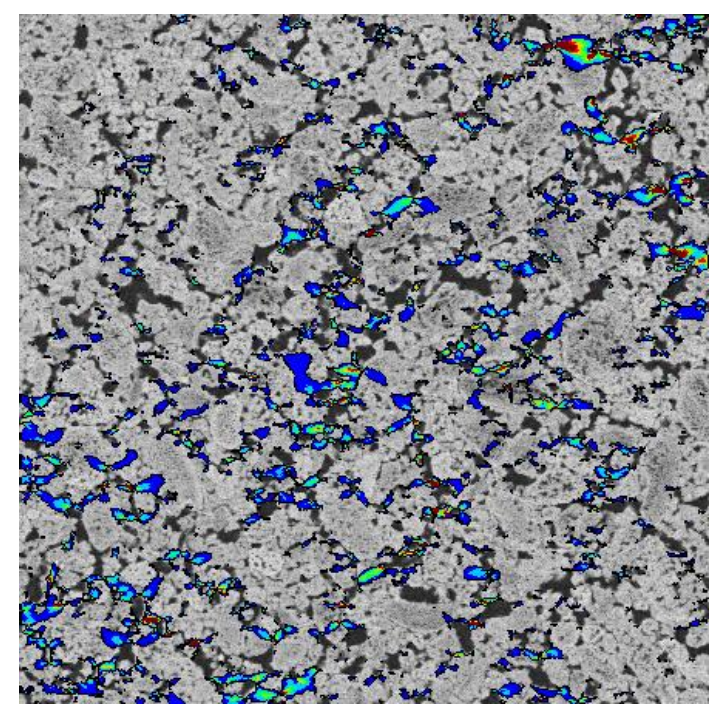

Figure 7 : a) SEM picture of the microstructure $(200 \mu \mathrm{m} \times 200 \mu \mathrm{m})$ b) Equivalent plastic strain field for $\Sigma_{11}=-20 \mathrm{MPa}$ superimposed on the microstructure 


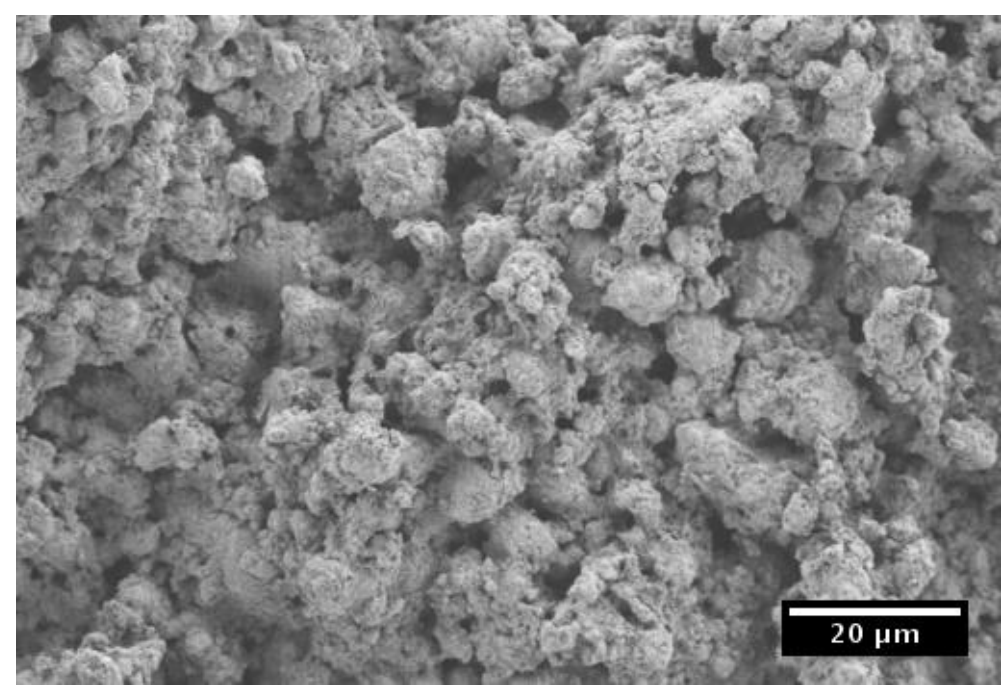

Figure 8 : SEM observation of macroporous material fracture surface (3-points bending test) : evidence of inter-granular fracture

\subsubsection{Estimation of the ductile damage criterion under compressive loads}

Figure 9 presents the plastic behavior of the macroporous material under compressive loads for several triaxiality ratios. The ductile behavior is emphasized by removing the elastic strains and plotting $\Sigma_{\mathrm{m}}\left(\mathrm{E}_{\mathrm{vol}}^{\mathrm{pl}}\right)$ and $\Sigma_{\mathrm{eq}}\left(\mathrm{E}_{\mathrm{eq}}^{\mathrm{pl}}\right)$. Whereas the matrix behavior is perfectly plastic, the macroporous material behavior is characterized by a significant strain hardening. Nevertheless, the plastic stiffness of the RVE at the final state is not zero. Otherwise, like under uniaxial compression, the mechanical state at onset of plastic damage is driven few elements at the extremities of pores branches. In order to estimate a ductile damage criterion, the critical mechanical state in each load case was conveniently stated to correspond to a common plastic dissipation energy $E_{P D}$ of $15 \mu \mathrm{J} / \mathrm{mm}^{3}$. This reference value has been identified experimentally under uniaxial compression as the approximate dissipation before brittle fracture.

The mechanical states corresponding to this reference plastic dissipation are reported in the $\left(\Sigma_{\mathrm{m}}, \Sigma_{\text {eq }}\right)$ plane, Figure 10. The locus thus defined forms an estimation of the ductile damage criterion that can be compared with the experimental results. The mesoporous material ductile damage criterion, i.e. the matrix criterion, is also recalled in Figure 10. Under negative mean stress, the criterion forms a closed surface, characterized by a cohesion around $17 \mathrm{MPa}$ and an hydrostatic yield pressure of $25 \mathrm{MPa}$. The experimental and numerical ductile damage criteria display a slightly different shape, but the amplitudes are very close. The uniaxial compression strength and hydrostatic yield pressure are in good agreement with the experimental values. 


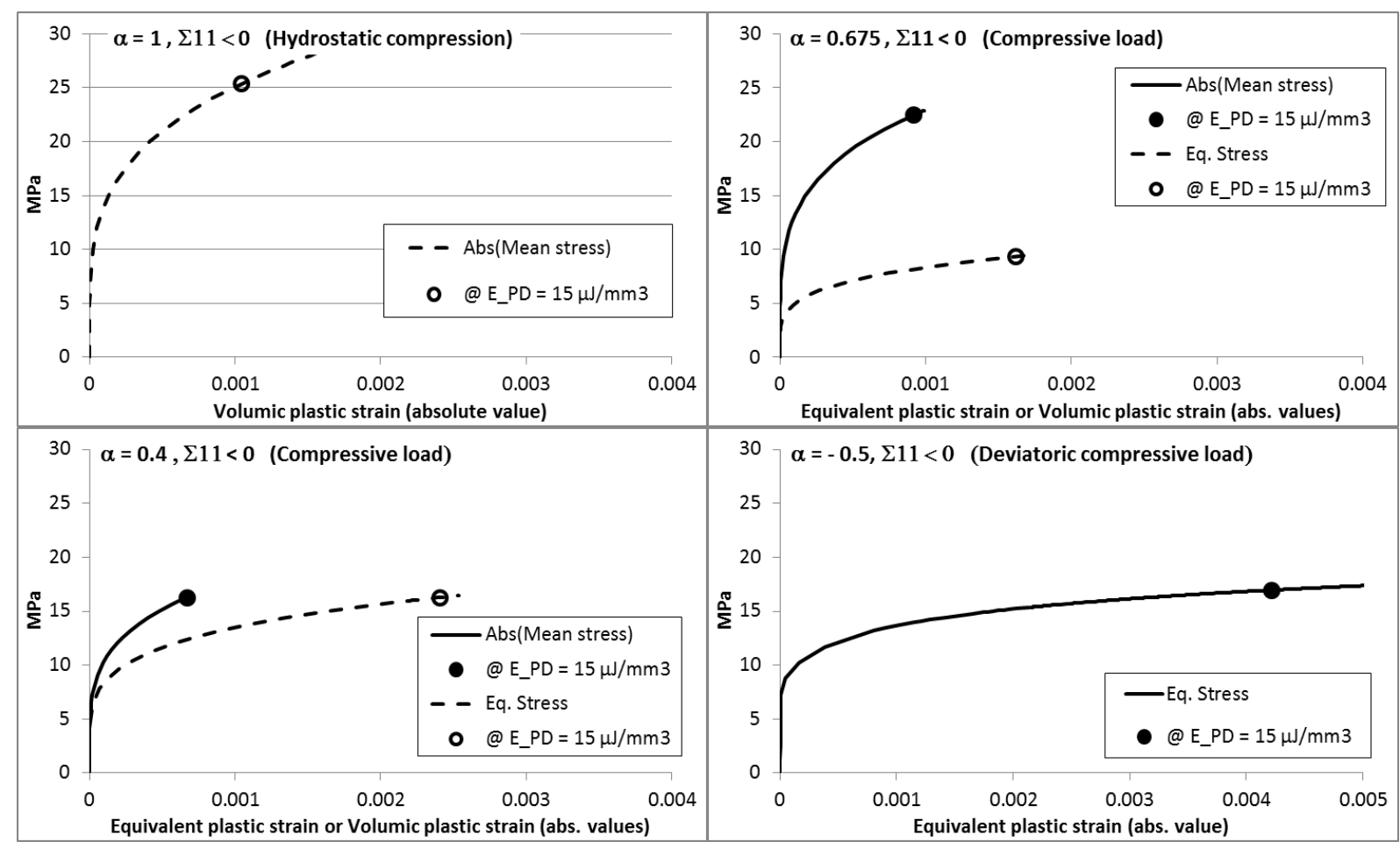

Figure 9 : Simulated macroscopic behavior of the macroporous material for different triaxiality ratio under compression : equivalent von Mises stress as a function of equivalent plastic strain and absolute mean stress as a function of volumic plastic strain, points indicate the mechanical state for a plastic dissipation energy of $E_{P D}=15 \mu \mathrm{J} / \mathrm{mm}^{3}$

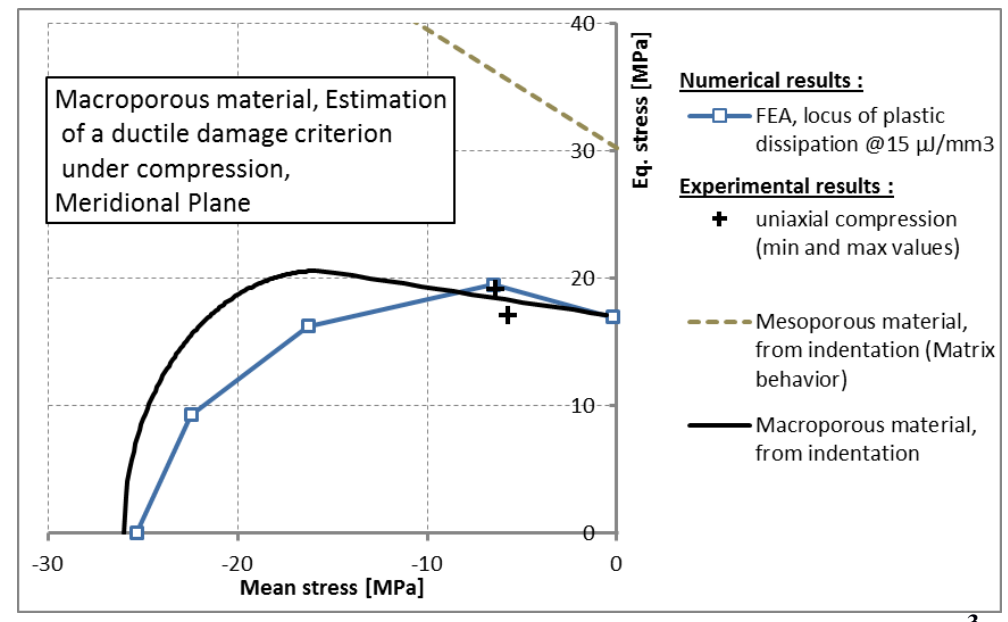

Figure 10 : Comparison of the locus of iso-plastic dissipation at $15 \mu \mathrm{J} / \mathrm{mm}^{3}$ obtained by the numerical model with experimental ductile damage criteria of the macroporous material. Mesoporous material ductile damage criterion determined experimentally and used in the simulation is also reported. Experimental results from (Staub et al., 2016)

\subsection{Ductile behavior under tensile loads $(\dot{\Sigma}>0)$}

The simulation did not intend to be representative of the experimental behavior under tensile stresses, as brittle-like mechanisms which were not included are rather favored in this domain by comparison with plastic mechanisms under compression. At that time, early divergence occurred because of localizations of plastic strain. Because of these limitations, the estimation of the ductile damage criterion was not extended under tensile loads. However, it is interesting to analyze the impact of the ductile damage mechanism in a wider range of stress triaxiality than it is available in the experimental database. A arbitrary plastic dissipation of $2 \mu \mathrm{J} / \mathrm{mm}^{3}$, close to the maximum dissipation available in tension loading cases, is chosen to plot the locus of the mechanical states, Figure 11. These 
mechanical states are characterized by a tensile stress $\Sigma_{11}$ close to $6 \mathrm{MPa}$, whatever the transverse stress $\Sigma_{22}$. It also corresponds to an affine relation between the equivalent stress and the mean stress.

Local plastic strain field confirms that plasticity activates under uniaxial tension in the same stress concentration zones as under uniaxial compression, drawing the same pattern of inter-granular damage. But plastic strains localization is higher : as shown in Figure 12, the local equivalent plastic strains exceeds $0.1 \%$ for a macroscopic tensile stress of only $6 \mathrm{MPa}$.

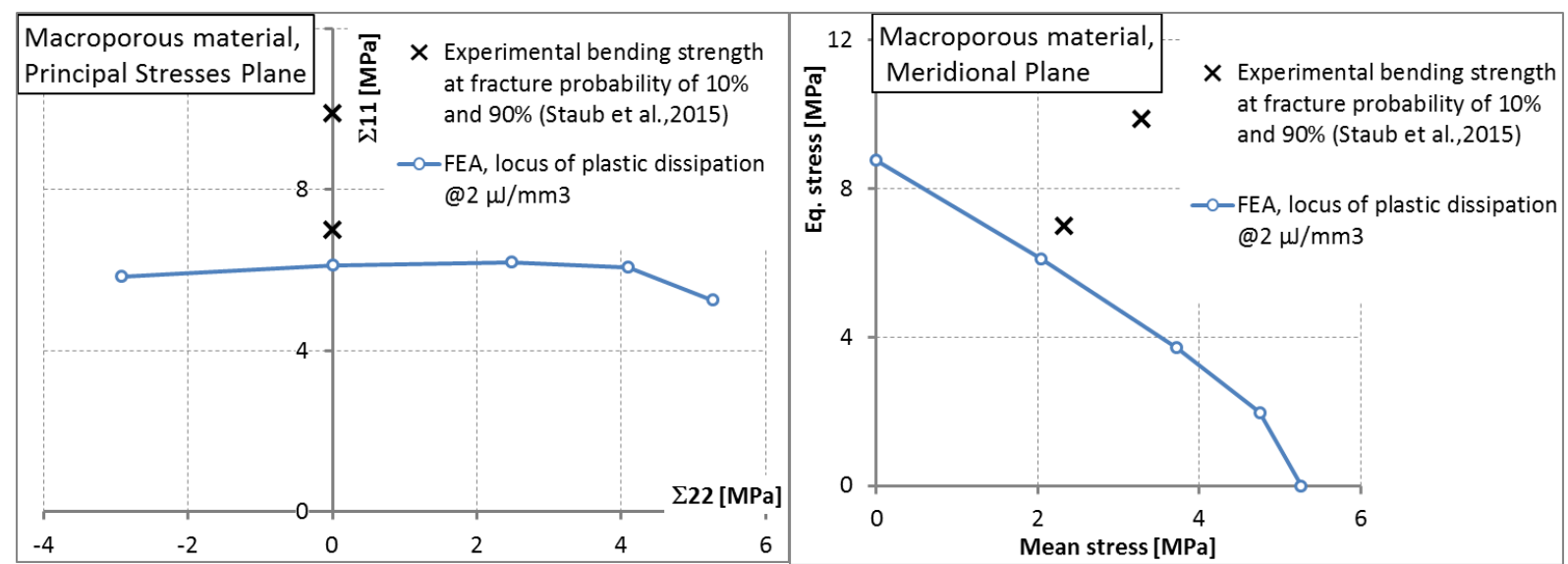

Figure 11 : Comparison of the locus of iso-plastic dissipation at $2 \mu \mathrm{J} / \mathrm{mm}^{3}$ obtained by the numerical model with experimental bending strength of the macroporous material

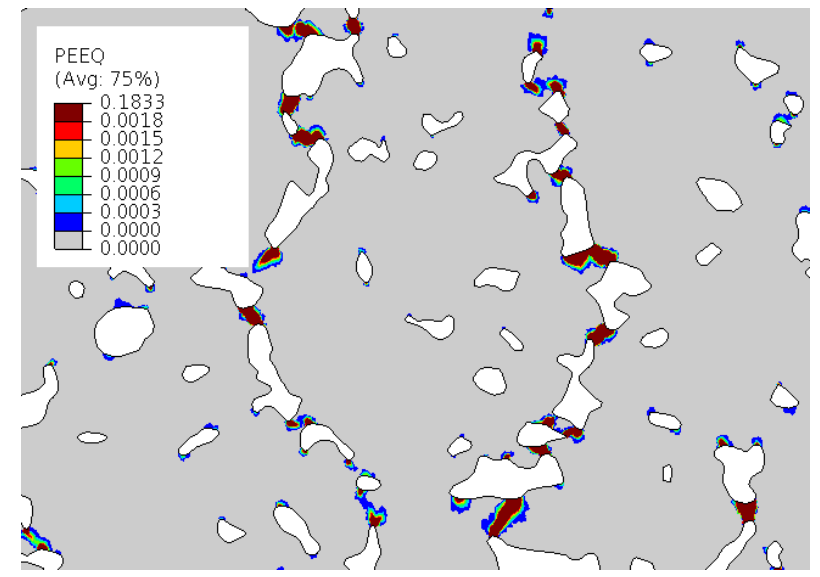

Figure 12 : Equivalent plastic strain field under uniaxial tension at end of simulation for $\Sigma_{11}=6 \mathrm{MPa}$

\section{Discussion}

Influence of the microstructure geometry

The interest of the digital image based approach is that the complexity of pore shapes is easily taken into account. Nevertheless, the pore contours extracted from digital image needed smoothing. This treatment was justified by higher resolution observations of pores shapes. It aimed at improving the relevance of the geometry before meshing. Stress concentration and plastic strain localization would be over-estimated if unrealistic sharp angles were retained. However, the influence the smoothing method on the results can be questioned, particularly under tensile loads where the early divergence occurred because of excessive localizations of plastic strain.

The use of a morphological model of the microstructure is an alternative approach that can advantageously be extended to 3D models. Granular porous medium could be represented by multiscale Boolean stacking of spheres with exponential size distribution (Jeulin, 2012). This model requires a fine adjustment of parameters on $2 \mathrm{D}$ images properties. With non-convex and branched porosities, the influence of the adjustment quality on the simulation results can remain an issue . 
Influence of the plastic criterion on the RVE failure process stability

Experimental evidence of irreversible and stable deformations in macro-porous alumina have been given by (Staub et al., 2016) under compressive loads. Progressive pores closure have been observed under indentation tests and under pure hydrostatic pressure tests without macroscopic cracking in the mesoporous matrix. Micro-cracking and frictional sliding between polycrystals agglomerates were suspected. A progressive stiffness decrease has also been observed under uniaxial compression before unstable macro crack propagation. At the macroscopic point of view, a behavior transition is thus observed : ductility appears under compression and increases with stress triaxiality. This transition can be due to the competition between two damage mechanisms, a ductile mechanism under compression and a brittle mechanism under tension. Or, the transition can be due to the same local damage mechanism that generates ductility under compression and unstable crack growth under tension. In the present study, a unique ductile damage criterion was considered to represent the local damage of the matrix, whatever the local triaxiality ratio. Plasticity concept was used to represent irreversible phenomenon like micro-cracking and by frictional sliding of the micro-crack surfaces. As a consequence, a stable growth of damaged zones was observed in the macroporous material, as seen in Figure 6. In case of robust convergence of the simulation, a complete failure of the RVE would be expected from the formation of a plastic path through the entire domain and coalescence of pores.

In the future, a brittle damage criterion would be worth being introduced in the model in order to reproduce the behavior transition and to investigate the influence of stress triaxiality on ductility. For instance, cohesive zones (Perales et al., 2008) could be used to simulate a brittle mechanism. A better understanding and characterization of the brittle damage mechanisms of the mesoporous matrix is necessary to formulate such a criterion. Experimental works are currently undertaken to address this issue.

Choice of a critical mechanical state to estimate the ductile damage criterion

The damage criterion estimated from simulations depends on the choice of a critical mechanical state. The plastic dissipation has been used in the definition of internal damage variables in early continuum damage models, e.g. (Lubliner et al., 1989). In homogeneous materials, the onset of plastic dissipation attests that the yield surface has been reached. In the case of a porous material, the mechanical state at onset of plastic damage corresponds to local plasticity at extremities of pores branches that is not representative of the RVE failure. Otherwise, the mechanical state at the end of the simulations overestimates the critical state of the RVE at failure because of the stability obtained with the local plastic criterion. The reference plastic dissipation energy chosen in the present study to estimate a ductile criterion corresponds to an intermediate state of the RVE. Nevertheless, the selected value has low influence on the shape of the criterion. As already obtained on a hollow sphere by analytical approach (Thoré et al., 2009) or by limit analysis in FEM (Pastor et al., 2013), we observe that the yield surface of a porous Drucker-Prager matrix with branched and non-convex pores is closed by a cap. The overall yield surface is qualitatively the same cap-form as those obtained in literature for isotropic porous media containing spherical voids (e;g; Shen et al., 2015), which seems to show that the pore shape does not strongly affect this cap-form since the overall isotropy is respected.

\section{Influence of a continuous representation of the matrix}

The porous granular material was simulated as a porous medium without representation of grains and their interfaces. Thus, the porous medium damage criterion does not distinguish grains strength from the interface strength. The simulation results showed that damage naturally initiates at pores extremities in stress concentration zones. The plastic damage propagates from pore to pore and thus follows paths close to grains interfaces, although interfaces were not explicitly represented in the simulation. The good accordance of this numerical analysis with experimental observations confirms such an inter-granular damage mechanism. The interface strength seems to be close to the grains strength, since no distinction was required to estimate the macroscopic strength of the granular medium. 


\section{Conclusions}

A numerical analysis of the ductile damage behavior of a macroporous $\gamma$-alumina was successfully carried out thanks to a full-field homogenization approach based on a FE model whose microstructure geometry was extracted from SEM images. The branched and non-convex shapes of pores were closely transcribed. A generalized plain strains formulation allowed to control the out-of-plane macrostress. The matrix behavior was considered elastic-plastic and followed a Drucker-Prager yield surface identified experimentally in a previous work on a mesoporous alumina. The mechanical properties of the macroporous material are numerically investigated in a wider range of stress triaxiality than it was available in the experimental database.

The model satisfactorily reproduces the progressive loss of stiffness observed experimentally under compression. Final rupture by macro-crack initiation and propagation in the RVE is not simulated. A reference plastic energy dissipation adjusted on uniaxial compression tests is used to estimate a damage criterion. Under compressive loads, the criterion obtained numerically is close to the closedform observed experimentally. The overall yield surface is qualitatively the same cap-form as those obtained in literature for isotropic porous media containing spherical voids, which seems to show that the pore shape does not strongly affect this cap-form since the overall isotropy is respected.

Under uniaxial traction or compression, the analysis of plastic strain fields showed localizations in areas that can be compared to grains interfaces, which is consistent with the inter-granular fracture revealed experimentally. Damage naturally localized in grains interfaces because of the morphology of the porosity. The morphology of the macropores is thus the root cause of degradation of the mechanical properties of the granular medium. This result leads to new opportunities in material design, for instance by controlling porosity shape thanks to pore forming agent. Mechanical properties of future materials might be anticipated by FE simulations based on optimized porosity morphology.

\section{Acknowledgments and funding}

This work was supported by IFP Energies nouvelles. The authors convey their sincere thanks to Dr. Sylvain Meille (MATEIS, INSA Lyon) for proof reading the article and to Dr. Laurent Cangémi (IFP Energies nouvelles) for fruitful discussions.

\section{References}

Ashby, M.F., Sammis, C.G., 1990. The damage mechanics of brittle solids in compression. pure and applied geophysics 133 (3), 489-521. 10.1007/BF00878002.

Ayyar, A., Chawla, N., 2006. Microstructure-based modeling of crack growth in particle reinforced composites. Composites Science and Technology 66 (13), 1980-1994. 10.1016/j.compscitech.2006.01.007.

Barthélémy, J.-F., Dormieux, L., 2003. Détermination du critère de rupture macroscopique d'un milieu poreux par homogénéisation non linéaire. Comptes Rendus Mécanique 331 (4), 271-276. 10.1016/S1631-0721(03)00063-9.

Bartuli, C., Bemporad, E., Tulliani, J.M., Tirillò, J., Pulci, G., Sebastiani, M., 2009. Mechanical properties of cellular ceramics obtained by gel casting: Characterization and modeling. Journal of the European Ceramic Society 29 (14), 2979-2989. 10.1016/j.jeurceramsoc.2009.04.035.

Bornert, M., Bretheau, T., Gilormini, P. (Eds.), 2001. Homogénéisation en mécanique des matériaux. Hermès Science publications, Paris, 255 pp.

Chawla, N., Deng, X., 2005. Microstructure and mechanical behavior of porous sintered steels. Materials Science and Engineering: A 390 (1-2), 98-112. 10.1016/j.msea.2004.08.046.

Dancette, S., Browet, A., Martin, G., Willemet, M., Delannay, L., 2016. Automatic processing of an orientation map into a finite element mesh that conforms to grain boundaries. Modelling and Simulation in Materials Science and Engineering 24 (5), 55014. 10.1088/0965-0393/24/5/055014.

Dirrenberger, J., Forest, S., Jeulin, D., 2014. Towards gigantic RVE sizes for 3D stochastic fibrous networks. International Journal of Solids and Structures 51 (2), 359-376. 10.1016/j.ijsolstr.2013.10.011. 
Drucker, D.C., 1966. The continuum theory of plasticity on the macroscale and the microscale. J.Materials 1 (4), 873-910.

Duckworth, W., 1953. Discussion of Ryshkewitch Paper by Winston Duckworth*. J American Ceramic Society 36 (2), 68. 10.1111/j.1151-2916.1953.tb12838.x.

El Moumen, A., Kanit, T., Imad, A., Minor, H.E., 2013. Effect of overlapping inclusions on effective elastic properties of composites. Mechanics Research Communications 53, 24-30. 10.1016/j.mechrescom.2013.07.007.

Fritzen, F., Forest, S., Böhlke, T., Kondo, D., Kanit, T., 2012. Computational homogenization of elasto-plastic porous metals. International Journal of Plasticity 29, 102-119. 10.1016/j.ijplas.2011.08.005.

Guo, T.F., Faleskog, J., Shih, C.F., 2008. Continuum modeling of a porous solid with pressuresensitive dilatant matrix. Journal of the Mechanics and Physics of Solids 56 (6), 2188-2212. 10.1016/j.jmps.2008.01.006.

Hazanov, S., Huet, C., 1994. Order relationships for boundary conditions effect in heterogeneous bodies smaller than the representative volume. Journal of the Mechanics and Physics of Solids 42 (12), 1995-2011. 10.1016/0022-5096(94)90022-1.

Jauffrès, D., Martin, C.L., Lichtner, A., Bordia, R.K., 2012. Simulation of the toughness of partially sintered ceramics with realistic microstructures. Acta Materialia 60 (12), 4685-4694. 10.1016/j.actamat.2012.05.024.

Jeulin, D., 2001. Caracterisation morphologique et modeles de structures aleatoires, in: Bornert, M., Bretheau, T., Gilormini, P. (Eds.), Homogénéisation en mécanique des matériaux. Hermès Science publications, Paris, pp. 95-132.

Jeulin, D., 2012. Morphology and effective properties of multi-scale random sets: A review. Comptes Rendus Mécanique 340 (4-5), 219-229. 10.1016/j.crme.2012.02.004.

Keleş, Ö., García, R.E., Bowman, K.J., 2013. Deviations from Weibull statistics in brittle porous materials. Acta Materialia 61 (19), 7207-7215. 10.1016/j.actamat.2013.08.025.

Khdir, Y.-K., Kanit, T., Zaïri, F., Naït-Abdelaziz, M., 2014. Computational homogenization of plastic porous media with two populations of voids. Materials Science and Engineering: A 597, 324-330. 10.1016/j.msea.2013.12.095.

Lowell, S., Shields, J.E., 1991. Interpretation of mercury porosimetry data, in: Powder Surface Area and Porosity, vol. 2. Springer-Verlag,Heidelberg, pp. 99-120.

Lubliner, J., Oliver, J., Oller, S., Oñate, E., 1989. A plastic-damage model for concrete. International Journal of Solids and Structures 25 (3), 299-326. 10.1016/0020-7683(89)90050-4.

Maalej, Y., Dormieux, L., Sanahuja, J., 2009. Micromechanical approach to the failure criterion of granular media. EUROPEAN JOURNAL OF MECHANICS A-SOLIDS 28 (3), 647-653. 10.1016/j.euromechsol.2008.10.010.

Matheron, G., 1972. Random sets theory and its applications to stereology. Journal of Microscopy 95 (1), 15-23. 10.1111/j.1365-2818.1972.tb03708.x.

Meille, S., Lombardi, M., Chevalier, J., Montanaro, L., 2012. Mechanical properties of porous ceramics in compression: On the transition between elastic, brittle, and cellular behavior. Journal of the European Ceramic Society 32 (15), 3959-3967. 10.1016/j.jeurceramsoc.2012.05.006.

Michel, J.C., Moulinec, H., Suquet, P., 2001. A computational scheme for linear and non-linear composites with arbitrary phase contrast. International Journal for Numerical Methods in Engineering 52 (12), 139-160. 10.1002/nme.275.

Miled, K., Le Roy, R., Sab, K., Boulay, C., 2004. Compressive behavior of an idealized EPS lightweight concrete: size effects and failure mode. Mechanics of Materials 36 (11), 1031-1046. 10.1016/j.mechmat.2003.08.004.

Moreaud, M., Cokelaer, F., 2015. Flowing Bilateral Filter: Definition and Implementations. Image Analysis \& Stereology 34 (2), 101. 10.5566/ias.1225.

Moulinec, H., Suquet, P., 1998. A numerical method for computing the overall response of nonlinear composites with complex microstructure. Computer Methods in Applied Mechanics and Engineering 157 (1-2), 69-94. 10.1016/S0045-7825(97)00218-1.

Otsu, N., 1979. A Threshold Selection Method from Gray-Level Histograms. IEEE Transactions on Systems, Man and Cybernetics 9 (1), 62-66. 10.1109/tsmc.1979.4310076. 
Pastor, F., Kondo, D., Pastor, J., 2013. Limit Analysis and computational modeling of the hollow sphere model with a Mises-Schleicher matrix. International Journal of Engineering Science 66-67, 60-73. 10.1016/j.ijengsci.2013.02.001.

Perales, F., Bourgeois, S., Chrysochoos, A., Monerie, Y., 2008. Two field multibody method for periodic homogenization in fracture mechanics of nonlinear heterogeneous materials. Engineering Fracture Mechanics 75 (11), 3378-3398. 10.1016/j.engfracmech.2007.07.017.

Petit, C., Meille, S., Maire, E., 2013. Cellular solids studied by x-ray tomography and finite element modeling - a review. Journal of Materials Research 28 (17), 2191-2201. 10.1557/jmr.2013.97.

Rice, R.W., 1993. Comparison of stress concentration versus minimum solid area based mechanical property-porosity relations. Journal of Materials Science 28 (8), 2187-2190. 10.1007/BF00367582.

Rudnicki, J.W., Rice, J.R., 1975. Conditions for the localization of deformation in pressure-sensitive dilatant materials. Journal of the Mechanics and Physics of Solids 23 (6), 371-394. 10.1016/00225096(75)90001-0.

Sanahuja, J., Dormieux, L., Meille, S., Hellmich, C., Fritsch, A., 2010. Micromechanical Explanation of Elasticity and Strength of Gypsum: From Elongated Anisotropic Crystals to Isotropic Porous Polycrystals. Journal of Engineering Mechanics 136 (2), 239-253. 10.1061/(ASCE)EM.19437889.0000072.

Shen, H., Brinson, L.C., 2007. Finite element modeling of porous titanium. International Journal of Solids and Structures 44 (1), 320-335. 10.1016/j.ijsolstr.2006.04.020.

Shen, W.Q., Shao, J.F., Kondo, D., Saxcé, G. de, 2015. A new macroscopic criterion of porous materials with a Mises-Schleicher compressible matrix. European Journal of Mechanics - A/Solids 49, 531-538. 10.1016/j.euromechsol.2014.09.010.

Staub, D., 2014. Étude du comportement mécanique à rupture des alumines de forte porosité: Application aux supports de catalyseurs d'hydrotraitement des résidus. Thèse de doctorat, Lyon.

Staub, D., Meille, S., Le Corre, V., Chevalier, J., Rouleau, L., 2015. Revisiting the Side Crushing Test Using the Three-Point Bending Test for the Strength Measurement of Catalyst Supports. Oil \& Gas Science and Technology - Revue d'IFP Energies nouvelles 70 (3), 475-486. $10.2516 /$ ogst $/ 2013214$.

Staub, D., Meille, S., Le Corre, V., Rouleau, L., Chevalier, J., 2016. Identification of a damage criterion of a highly porous alumina ceramic. Acta Materialia 107, 261-272. 10.1016/j.actamat.2016.01.071

Thoré, P., Pastor, F., Pastor, J., Kondo, D., 2009. Closed-form solutions for the hollow sphere model with Coulomb and Drucker-Prager materials under isotropic loadings. Comptes Rendus Mécanique 337 (5), 260-267. 10.1016/j.crme.2009.06.030.

Wagh, A.S., Singh, J.P., Poeppel, R.B., 1993. Dependence of ceramic fracture properties on porosity. Journal of Materials Science 28 (13), 3589-3593. 10.1007/BF01159841. 